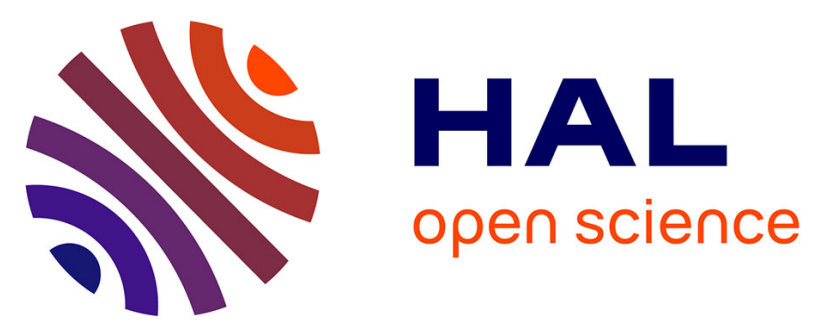

\title{
INDOCYANINE GREEN ANGIOGRAPHY FOR IDENTIFYING TELANGIECTATIC CAPILLARIES IN DIABETIC MACULAR EDEMA
}

Daniela Castro Farías, Rodrigo Matsui Serrano, Juan Bianchi Gancharov, Ulises de Dios Cuadras, José Sahel, Federico Graue Wiechers, Benedicte Dupas, Michel Paques

\section{To cite this version:}

Daniela Castro Farías, Rodrigo Matsui Serrano, Juan Bianchi Gancharov, Ulises de Dios Cuadras, José Sahel, et al.. INDOCYANINE GREEN ANGIOGRAPHY FOR IDENTIFYING TELANGIECTATIC CAPILLARIES IN DIABETIC MACULAR EDEMA. British Journal of Ophthalmology, 2020, 104 (4), pp.509-513. 10.1136/bjophthalmol-2019-314355 . hal-02995350

\section{HAL Id: hal-02995350 https://hal.sorbonne-universite.fr/hal-02995350}

Submitted on 9 Nov 2020

HAL is a multi-disciplinary open access archive for the deposit and dissemination of scientific research documents, whether they are published or not. The documents may come from teaching and research institutions in France or abroad, or from public or private research centers.
L'archive ouverte pluridisciplinaire HAL, est destinée au dépôt et à la diffusion de documents scientifiques de niveau recherche, publiés ou non, émanant des établissements d'enseignement et de recherche français ou étrangers, des laboratoires publics ou privés. 


\section{INDOCYANINE GREEN ANGIOGRAPHY FOR IDENTIFYING TELANGIECTATIC CAPILLARIES IN DIABETIC MACULAR EDEMA}

Daniela Castro-Farías ${ }^{1}$, Rodrigo Matsui-Serrano ${ }^{1}$, Juan Bianchi-Gancharov ${ }^{1}$, Ulises de Dios-Cuadras ${ }^{1}$, José-Alain Sahel ${ }^{2,3}$, Federico Graue-Wiechers ${ }^{1}$, Bénédicte Dupas ${ }^{4}$, Michel

$$
\text { Paques }^{2,3}
$$

From the ${ }^{1}$ Instituto de Oftalmología Conde de Valenciana, Mexico City, Mexico, ${ }^{2}$ the Quinze-Vingts hospital, DHU Sight Maintain, INSERM-DHOS CIC 1423, Paris, F-75012, France; ${ }^{3}$ Sorbonne Université, Paris, France; ${ }^{4}$ the Hopital Lariboisière, Assistance-Publique Hopitaux de Paris, Paris, France

Conflict of interest: none

Supported by the Agence Nationale de la Recherche (ANR-14-CE17-0011-01), the Association Contre l'OVR (www.asso-ovr.fr), the Programme Hospitalier de Recherche Clinique (TalaDMEproject PHRC-N-17-0334), the French Clinical Research Network, the Institut Hospitalo-Universitaire ForeSight and the Comité National Coordination Action Handicap. The funding organizations had no role in the design or conduct of this research.

Correspondance: Michel Paques, MD, PhD, Quinze-Vingts hospital, 28 rue de Charenton, Paris F-75012, France.Tel +33 140021415 Fax + 33-1- 40021499 e-mail mpaques@15-20.fr

Keywords: Diabetic Macula Edema, Indocyanine Green Angiography, Microvascular Abnormalities, Hard Exudates 
Aims: During diabetic macular edema (DME), a spectrum of capillary abnormalities is commonly observed, ranging from microaneurysms to large microvascular abnormalities. Clinical evidence suggests that targeted photocoagulation of large microvascular abnormalities may be beneficial, but their detection is not done in a routine fashion. It was reported that they are better identified by indocyanine green angiography (ICGA) than by fluorescein angiography. Here, we investigated the prevalence and ICGA and OCT features of large microvascular abnormalities in a group of patients with DME.

Methods: Observational study. The fundus photographs, ICGA and structural and angiographic OCT charts of 35 eyes from 25 consecutive patients with DME were reviewed.

Results: Twenty-two eyes (63\%) had at least one focal area of microvascular abnormalities showing prolonged ICG staining (i.e. beyond $10 \mathrm{mn}$ after injection). In particular, all eyes with circinate hard exudates $(n=9)$ showed foci of late ICG staining in the center of exudates. These areas appeared either as isolated globular capillary ecstasies or as a cluster of ill-defined capillary abnormalities. They were located at a median distance of $2708 \mu \mathrm{m}$ from the fovea (range: $1064-4583 \mu \mathrm{m}$ ). Their diameter ranged from 153 to $307 \mu \mathrm{m}$. During ICGA, 94\% of such large lesions showed an increase in their contrast and apparent size in late frames relative to early frames, while $77 \%$ of microaneurysms showed reduced contrast on late frames. OCTA appeared as poorly contributive for the detection of ICG stained lesions.

Conclusion: Late ICG staining revealing large microvascular abnormalities is commonly observed during DME. Because of their specific angiographic and OCT features relative to microaneurysms, we propose to name them telangiectatic capillaries. 
The worldwide epidemic of diabetes challenges the sustainability of health care systems. ${ }^{1}$ Diabetes indeed leads to a variety of metabolic, vascular and neurological complications, among which vision loss is one of the most common as well as one of the most feared by patients. After cataract, macular edema is the second cause of visual loss in patients with diabetes. In Mexico the prevalence of diabetes is around $9.4 \%{ }^{2}$ with an estimated incidence of $8.8 \%$ of diabetic macular edema (DME). ${ }^{3}$

The canonical lesions of diabetic retinopathy are microaneurysms; however, the spectrum of retinal microvascular abnormalities also comprises large microvascular abnormalities ${ }^{4,5}$ which can reach a diameter of several hundred microns. ${ }^{6}$ These have been reported in a variety of retinal vascular diseases among which retinal vein occlusion, Coat's disease and macular telangiectasia. ${ }^{7-16}$ These lesions have received various terms in the literature, such as capillary macroaneurysms, ${ }^{6-10,13,16}$ adult Coats' disease ${ }^{11}$ or distant retinal vascular anomalies. ${ }^{14}$ Since the term "macroaneurysm" creates confusion with arterial macroaneurysms, that Coat's disease is clearly a misnomer, and that he term "distant" is inappropriate because these may be found in the macula as well, we will use here the generic term telangiectatic capillaries (TCs).

Following targeted photocoagulation of TCs, anatomical and visual improvement of macular edema has been reported, ${ }^{15,16}$ which supports the interest of their identification. To optimize their detection, of importance is to take into account the fact that they are better identified by indocyanine green angiography (ICGA) than by fluorescein angiography (FA). ${ }^{7,14-16}$ In particular, ICG staining often persist in late frames; additionally, ICGA seldom leaks out from these lesions which contributes to their high contrast. Improving the knowledge of their clinical characteristics may contribute to improve their identification. Here, in the continuity of previous studies, ${ }^{8,16}$ we further explored the ICGA and OCT features of vascular abnormalities of DME eyes. 


\section{METHODS}

This observational study was approved by the Internal Review Board of the Institute Conde de Valenciana. Each patient received oral and written information and gave consent for examination. Consecutive patients diagnosed with clinically significant DME according to the ETDRS criteria ${ }^{17}$ were identified. These criteria comprise (1) thickening of the retina within $500 \mu \mathrm{m}$ of the center of the macula; or (2) hard exudates (HEs) at or within $500 \mu \mathrm{m}$ of the center of the macula associated with thickening of adjacent retina; or (3) a zone of retinal thickening one disc area or larger, any part of which is within one disc diameter of the center of the macula. HEs were classified as grade 1 (dust-like HEs), grade 2 (aggregated HEs), or grade 3 (circinate disposition and/or foveal accumulation of aggregated HEs). Examples of each grade are shown in figure 1.
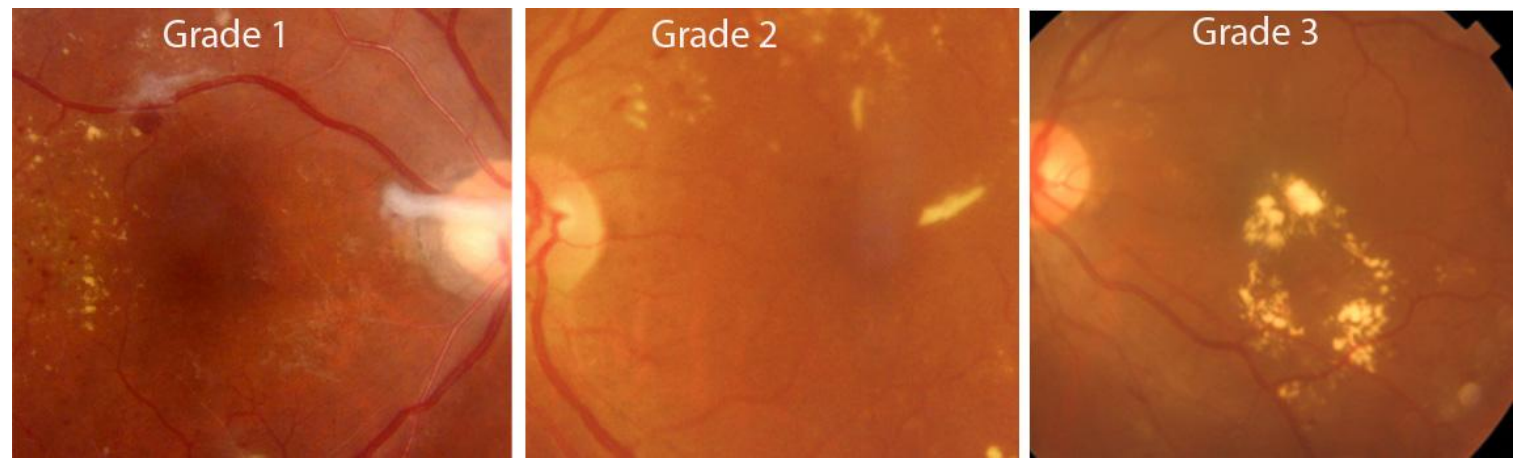

Figure 1. Grading of hard exudates (HEs) used in the present study.

ICGA was done using the Spectralis scanning laser ophthalmoscope-OCT (SLO-OCT; Heidelberg Engineering, Heidelberg, Germany) using $5 \mathrm{ml}$ of intravenous Infracyanine $\circledast$ (SERB laboratories, Paris, France). A particular attention was paid at obtaining late frames (i.e. at least 10 minutes after dye injection). As a rule, late frames were averaged using the built-in software to improve signal-to-noise ratio. The presence of TCs was evaluated by three experienced retinal specialists (DCF, BD, MP) based primarily on size and ICGA staining. Early, late and wash-out phases were defined as follows: early frames were those showing details of choroidal large vessels; late frames were those showing a uniform 
background fluorescence of the choroid, and wash-out frames were those showing retinal vessels darker than the background. Each microvascular lesion was classified as microaneurysms or TCs based on its size, assuming that microaneurysms were spherical lesions with an upper size limit of $130 \mu \mathrm{m} .{ }^{18,19}$ The size of the TCs were measured on ICGA images and on OCT using the built-in calipers. Structural OCT also enabled to measure the thickness of the wall of TCs.

OCT angiography (OCTA) images centered on the fovea $(6 \times 6 \mathrm{~mm}$ cubes) were obtained using a DRI Triton OCT device (Topcon, Tokyo, Japan) in a subset of patients. Each en OCT angiogram ICGA and OCTA images were reviewed in a masked fashion by two experienced retinologists (DCF and $\mathrm{MP})$.

\section{RESULTS}

Our series comprised 35 eyes with clinically significant DME from 25 type 2 diabetic patients (12 females, 13 males; mean age 61.2 years old). Visual acuities ranged from counting fingers to $20 / 20$ (median, 20/80). Nonproliferative diabetic retinopathy was present in 16 eyes (45.7\%), proliferative diabetic retinopathy in 6 eyes $(17.1 \%)$, and panretinal photocoagulation has been done previously in $13(37.1 \%)$. The mean $( \pm S D)$ central macular thickness was $282.4 \mu \mathrm{m}( \pm 145)$. Ten eyes had grade $1 \mathrm{HEs}, 14$ had grade 2 and 9 eyes had grade 3 HEs.

In early frames, all microaneurysms were filled by ICG, hence showing a bright contrast, and were most often indistinguishable from TCs. On subsequent frames, TCs typically increased both their apparent size and their contrast with the background, while microaneurysms rapidly reached their peak contrast and did not increase in size (figure 2 and supplementary figure 1). On late frames, 22 eyes (63\%) had at least one focal area of ICG staining corresponding to TCs. TCs were located at a median distance of $2708 \mu \mathrm{m}$ from the fovea (range: $1064-4583 \mu \mathrm{m}$ ). These areas showed variable aspects, from globular, solitary TCs (6 cases) to a cluster of irregularly shaped lesions (18 cases), some subjects 
having both. In two eyes, progressively increasing ICG leakage from a TC was seen on wash-out frames (figure 3).

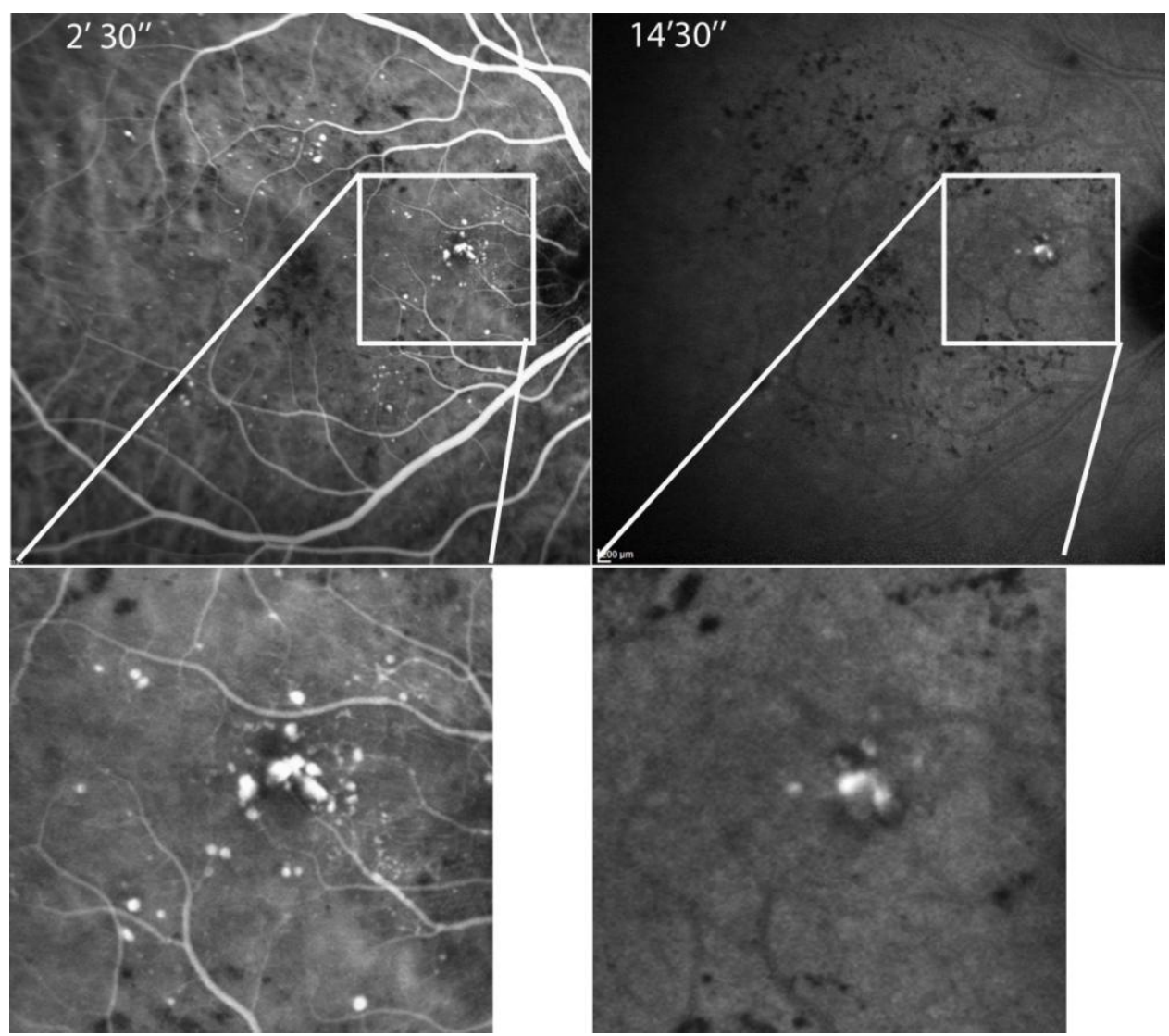

Figure 2. Comparison of early and late ICG staining patterns of microaneurysms and TCs (case 12). Time indicates duration since dye injection. Between the early and late frame, note the fading contrast of the microneurysm, while the TCs increase their contrast (see also supplementary figure 1). 

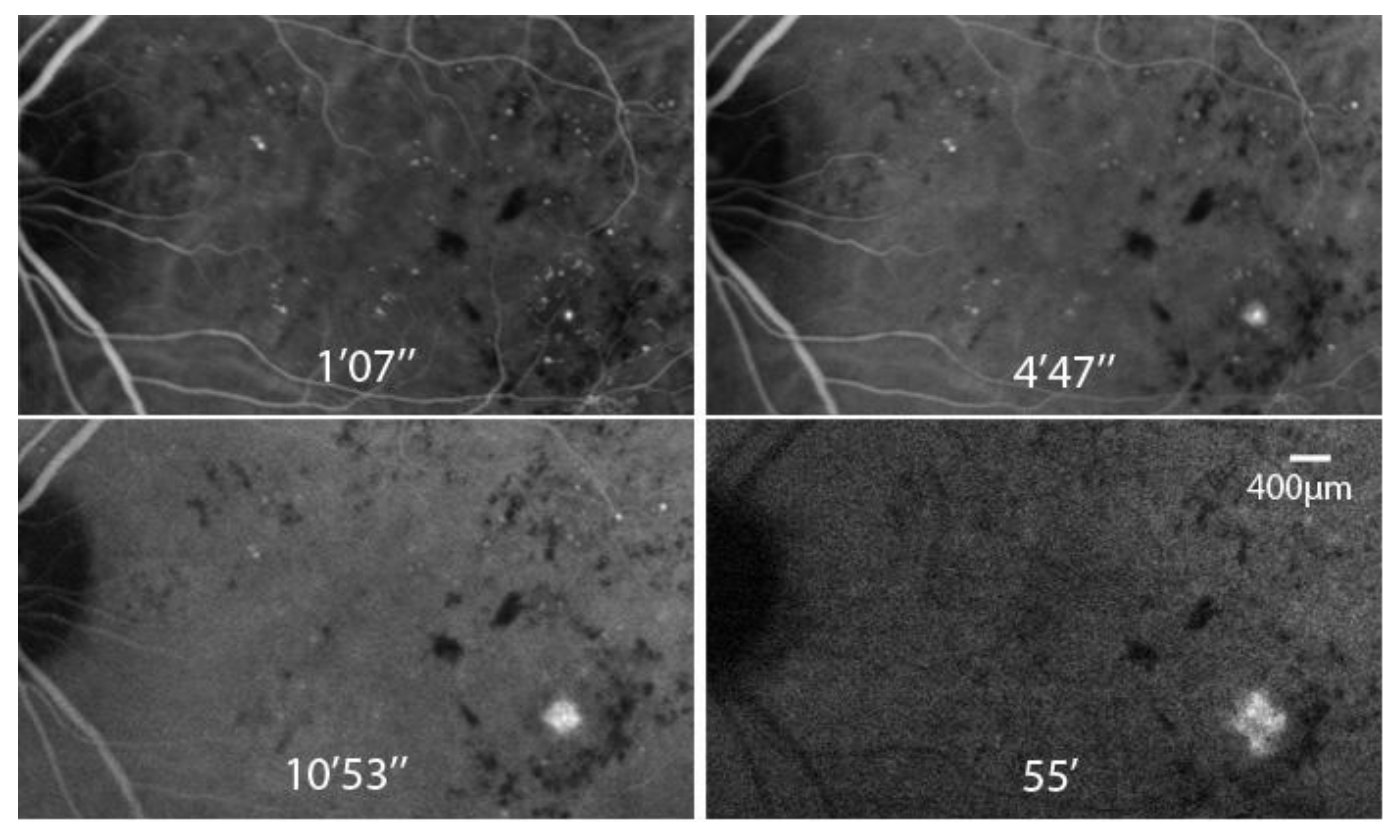

Figure 3. Case 4. Illustration of ICG leakage of TC on wash-out frames.

We compared the ICGA features of microaneurysms and TCs. Among 124 microaneurysms from 5 subjects, all were visible in early frames, but only $23 \%$ remained visible on late frames; On the opposite, among 23 TCs, $92 \%$ were better detected on late than early frames, because on smaller frames they appeared smaller or even not stained at all.

The median diameter of TCs was $227.5 \mu \mathrm{m}$ (range: 153-307 $\mu \mathrm{m}$ ). The thickness of the wall of globular TCs ranged from 8 to $154 \mu \mathrm{m}$ (median, $76 \mu \mathrm{m}$ ). The lumen of solitary TCs was in all cases filled with reflective material, while its presence was more difficult to assess in irregular TCs due to their smaller size. Clustered TCs showed variable contrast and, when considered individually, were often undistinguishable from microaneurysms. Examples of structural OCTs of TCs are shown in figure 4. 

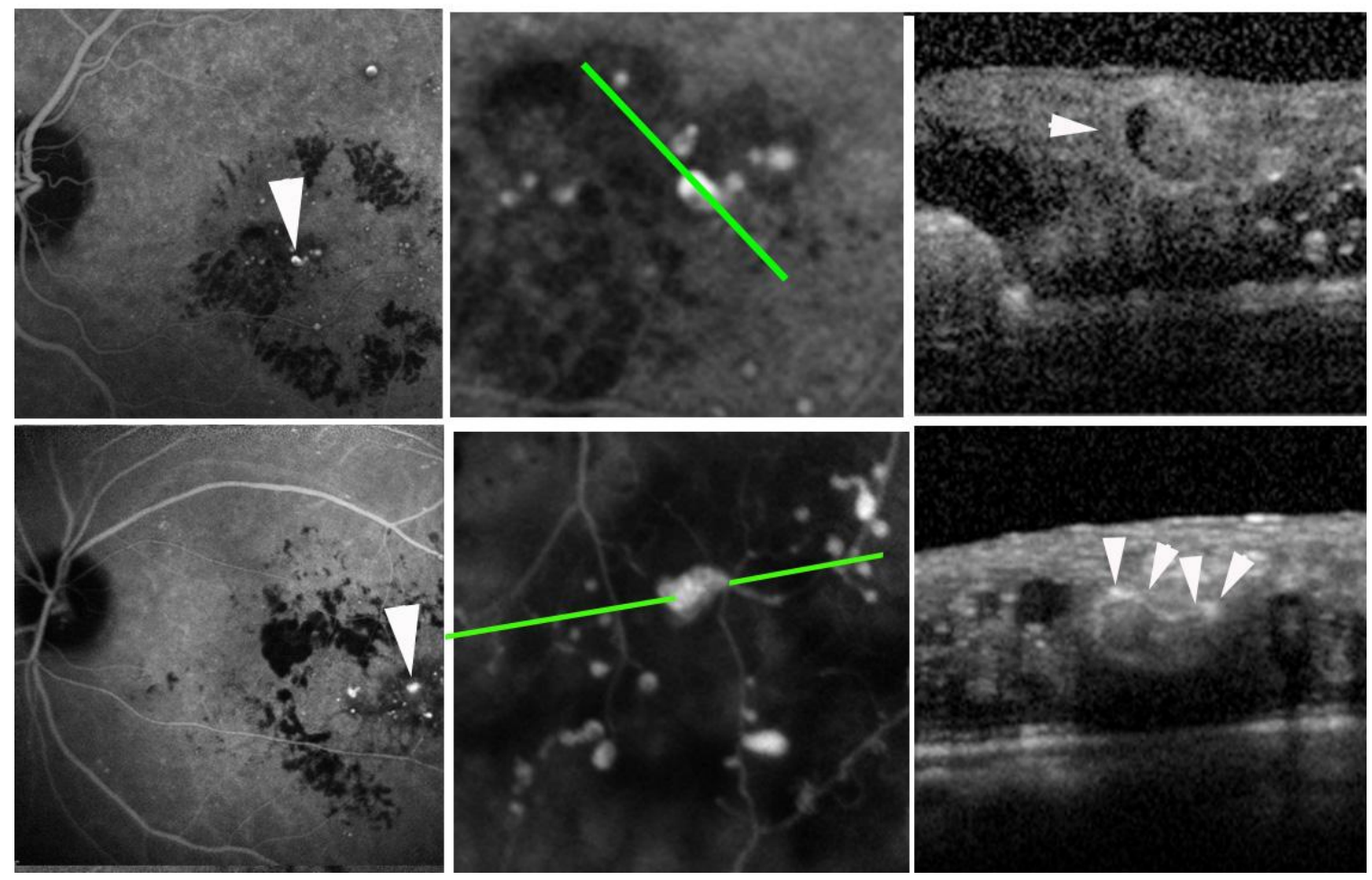

Figure 4. Illustration of different presentations of TCs (arrowheads) by ICGA and structural OCT.

Top row, case 10; bottom row, case 2. Note the presence of intraluminal material in the case shown in the top row.

We also analyzed the contribution of OCT angiography to the detection of TCs. Figure 5 shows the case of a very large TC which shows marked staining by ICG but poor contrast by OCTA. Overall, there was an inverse correlation between the size of TC and their detection by OCTA: the larger the TC, the less likely they were to be detected by OCTA. OCTA detected 2 out of 4 clustered TC but failed to detect 5 out of 5 globular TC. 

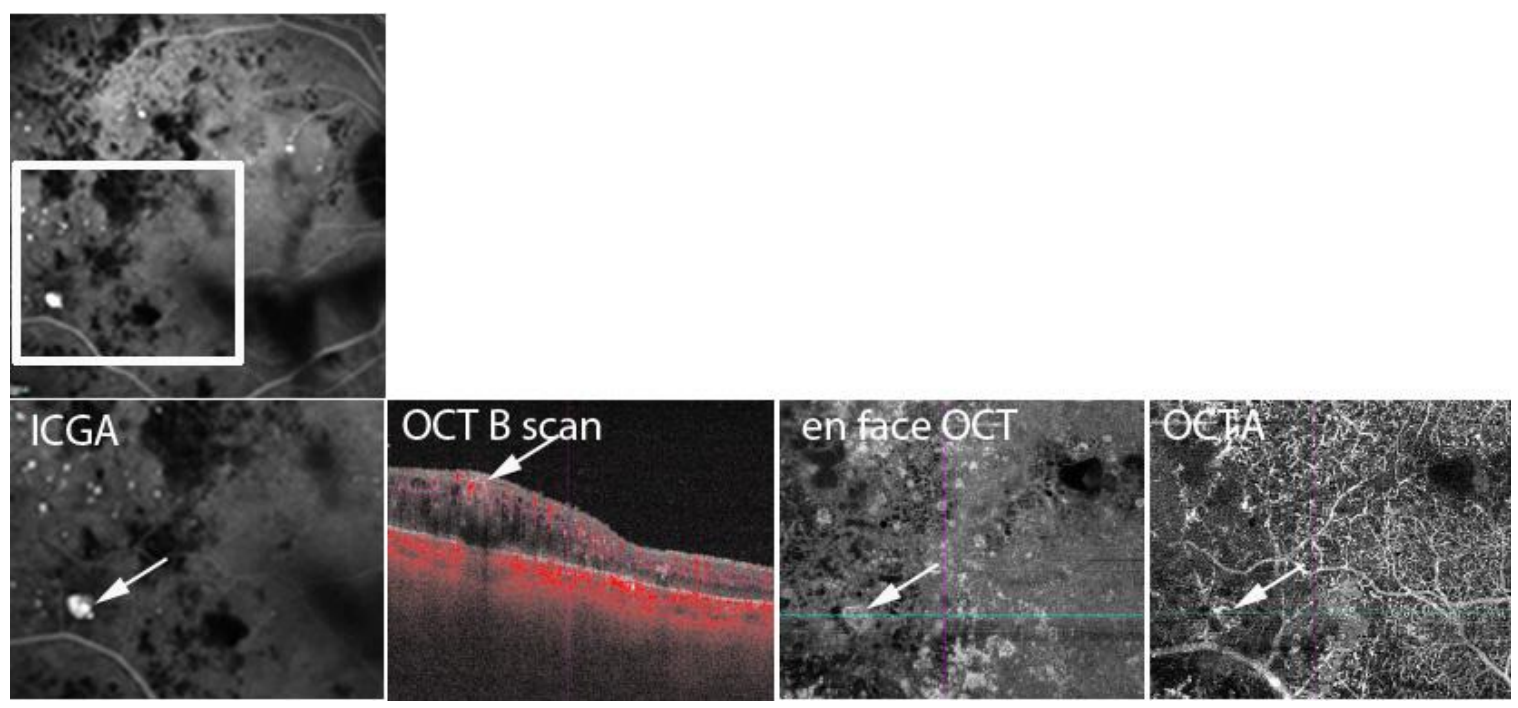

Figure 5. Case 25. Example of a TC (arrows in successive images) seen by ICGA, structural OCT and OCTA. Note that the TC is better detected by ICGA than by OCTA.

Among demographic and clinical characteristics (age, sex, duration of diabetes, macular thickness and severity of HEs), only the severity of HEs showed a statistically significant association with the presence of TCs. The prevalence of TCs indeed increased from 4/10 in eyes with grade $1 \mathrm{HEs}$, to $9 / 14$ in eyes with grade $2 \mathrm{HEs}$ and to all 9 eyes with grade $3 \mathrm{HEs}$ $\left(p=0.03 ; \mathrm{Chi}^{2}\right.$ test). Figure 6 shows a representative example of an eye with grade $3 \mathrm{HEs}$ with TCs in the geometrical center of circinate exudates (see also supplementary figure 1 and 2)

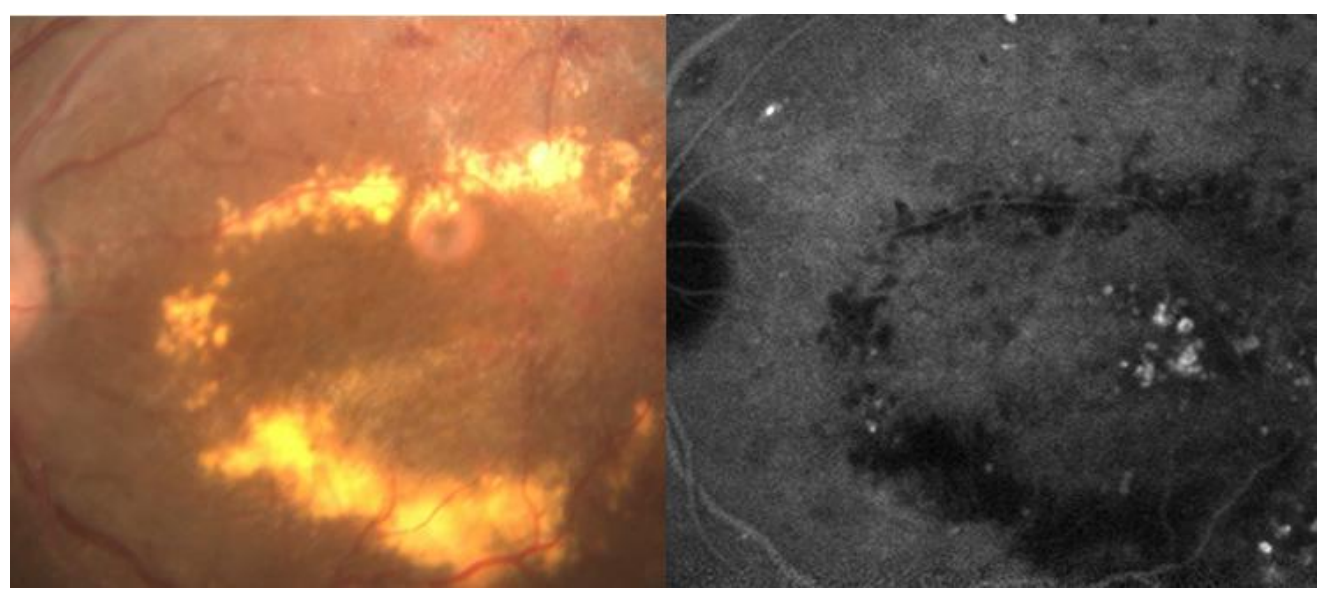

Figure 6. Case 5. Left, color fundus photograph showing circinate exudates. Right, late ICG frame showing clustered TC within the ring of exudates (see also supplementary figure 2). 


\section{Discussion}

During retinal vascular diseases a spectrum of microvascular changes may occur, the relationship of which with vascular function is poorly known. We have previously shown that while IGCA and FA both are useful to detect microaneurysms, ICGA is more efficient than FA to detect large microvascular lesions. The interest of ICGA is illustrated by the fact that all cases of circinate exudates showed foci of prolonged, elective ICG staining within them, strongly suggesting that late ICG staining is an indicator of severe rupture of the blood-retinal barrier. Since ICGA is not yet routinely done for the management of DME, many clinical features of TCs still remain to be documented. Here we report the prevalence and OCT and ICGA features of large and/or clustered microvascular abnormalities in a cohort of DME subjects. We found that nearly two third may show late, focal ICG staining of large microvascular abnormalities. Furthermore, we observed that, compared to "classical" microaneurysms, TCs had delayed filling as well as a delayed wash-out. This was more clearly apparent in the largest lesions, some of which retaining ICG even in wash-out frames, that is, up to one hour after dye injection. Overall, the larger the size of TCs, the more there is a temporal shift of ICG staining.

Accordingly, a report on adaptive optics ophthalmoscopy of microaneurysms have showed that some irregularly shaped microaneurysms are incompletely filled by fluorescein. ${ }^{20}$ It can be suggested that such incomplete filling may be due to the presence of intraluminal material as shown by OCT. Therefore, the delayed staining of TCs on ICGA that we observed may be due to the staining of intraluminal material. Histology studies suggested that the nature of this intraluminal material may be either stagnant blood, lipids and/or thrombosis, ${ }^{21}$ all of which may show affinity for ICG.

OCTA is gaining interest due to its capacity to noninvasively document the retinal vasculature, and had been reported to be able to document features of diabetic retinopathy with a sensitivity comparable to FA. However, OCTA documents the circulation of red cells 
rather than the structure of the microvascular anomalies themselves, hence the inherent limitations of OCTA to identify some of the largest TCs. Accordingly, we observed that ICGA and structural OCT were more efficient than OCTA to detect TCs because large TCs may be paradoxically undetectable by OCTA. The combination of wall thickening and intraluminal material accumulation provides a straightforward explanation for the reduced blood flow and hence the absence of detection of some of the largest TCs by OCTA.

The currently recommended first line treatment of DME is intravitreal treatment using either anti-VEGF or steroid. ${ }^{22-30}$ On the opposite, randomized trials on targeted laser photocoagulation of microaneurysms in DME did not reach their primary endpoint. ${ }^{31,32}$ It is interesting to note that in these trials only microvascular lesions identified by FA were included. Therefore, since FA is less sensitive than ICGA for the detection of TCs, large lesions may have been missed. These trials therefore leave open the question of the interest of photocoagulation of large lesions. Pilot studies of treatment of TCs by targeted photocoagulation has reported encouraging results ${ }^{6,14-16}$ yet a randomized trial remains to be done to confirm the efficacy of such procedure. Our results suggest that an optimal strategy aimed at detecting candidates for targeted photocoagulation implies performing ICGA. However, ICGA is somehow invasive, and also implementing ICGA for all DME patients may be challenging for the limited resources of many ophthalmological settings dealing with large populations of patients such as in developed countries. Therefore, identifying subgroups of patients with a high prevalence of TCs may be more pertinent. HEs are a hallmark of chronic macular edema. In the literature, among 24 cases reports of eyes with TCs of different etiologies, severe HEs were present in 11 cases $^{6-16}$ supporting a strong association between severe HEs and TCs. This is understandable since TCs are the site of a severe rupture of the blood-retinal barrier, enabling large molecular complexes such as lipoproteins to accumulate in the retina. In our series, the prevalence of TCs was correlated with the severity of HEs, peaking to $100 \%$ in presence of circinate HEs. Hence, the more severe the 
HEs, the more likely there is a TC, suggesting that the presence of severe HEs is a pertinent screening feature.

There are several limits to our study. There is yet no consensual definition of a TC, so their diagnosis may differ among operators. What we termed here TCs was defined primarily by a prolonged, focal ICG staining of a retinal vascular structure larger than a microaneurysm. In the near future cumulative clinical expertise and clinical trials may lead to a more rigorous definition of TCs, which may include structural features such as the thickness of the wall. We indeed observed that some TCs have a considerably thickened wall that may reach more than 100 microns. Another limitation was that our sample was small and comprised only type 2 diabetics with a high prevalence of proliferative diabetic retinopathy, possibly reflecting a poor glycemic control and/or other population-related factors. We had limited access to data on blood pressure and on serum lipids. Therefore, to which extent do these results apply to other populations remains to be determined.

We conclude that telangiectatic microvascular abnormalities showing late ICG staining are commonly observed in DME eyes. Given the fact that photocoagulation of these lesions may be beneficial for treating macular edema, it is of interest to screen eyes with severe DME for their presence. Comparing structural and angiographic OCT may also be of interest to identify photocoagulation targets since the presence of intraluminal material is indicative of BRB rupture.

\section{Acknowledgements}

Alvaro Rendón, Enrique Graue Hernández and David Lozano Rechy

\section{References}

1. Varma R, Bressler NM, Doan QV, et al. Prevalence of and risk factors for diabetic macular edema in the United States. JAMA Ophthalmol 2014;132:1334-40 
2. Rojas-Martínez R, Basto-Abreu A, Aguilar-Salinas CA, et al. Prevalence of previously diagnosed diabetes mellitus in Mexico. Salud Publica Mex. 2018;60:224-232.

3. González-Villalpando C, González-Villalpando ME, Rivera Martínez D, et al. Incidence and progression of diabetic retinopathy in low income population of Mexico City. Rev Invest Clin 1999;51:141-50.

4. Bresnick GH. Diabetic macular edema. A review. Ophthalmology 1986;93:989-97

5. Focal photocoagulation treatment of diabetic macular edema. Relationship of treatment effect to fluorescein angiographic and other retinal characteristics at baseline: ETDRS report no 19. Early Treatment Diabetic Retinopathy Study Research Group. Arch Ophthalmol 1995;113:1144-1155.

6. Bourhis A, Girmens JF, Boni S, et al. Imaging of macroaneurysms occurring during retinal vein occlusion and diabetic retinopathy by indocyanine green angiography and high resolution optical coherence tomography. Graefes Arch Clin Exp Ophthalmol 2010;248:161-6.

7. Schulman J, Jampol LM, Goldberg MF. Large capillary aneurysms secondary to retinal venous obstruction. Br J Ophthalmol 1981;65:36-41.

8. Cousins SW, Flynn HW Jr, Clarkson JG. Capillary macroaneurysms associated with retinal branch vein occlusion. Am J Ophthalmol 1990;109:567-70.

9. Parodi MB, Da Pozzo S, Saviano S, et al. Branch retinal vein occlusion and macroaneurysms. Int Ophthalmol 1997;21:161-4.

10. Battaglia Parodi M, Bondel E, Ravalico G. Capillary macroaneurysms in central retinal vein occlusion. Ophthalmologica 1995;209:248-50.

11. Luckie AP, Hamilton AM. Adult Coats' disease in branch retinal vein occlusion. Aust $N Z J$ Ophthalmo. 1994Aug;22:203-6.

12. Apostolopoulos M, Koutsandrea C, Chatjoulis D, et al. Late complications in branch retinal vein occlusion. Int Ophthalmol 1995-1996;19:281-5. 
13. Bastawrous A, Kumar V, Prasad S. Medical image. Retinal macroaneurysm following a branch retinal vein occlusion. N Z Med J 2011;124:103-5.

14. Ueda T, Gomi F, Suzuki M, et al. Usefulness of indocyanine green angiography to depict the distant retinal vascular anomalies associated with branch retinal vein occlusion causing serous macular detachment. Retina 2012;32:308-13

15. Ogura S, Yasukawa T, Kato A, et al. Indocyanine Green Angiography-Guided Focal Laser Photocoagulation for Diabetic Macular Edema. Ophthalmologica 2015;234:139-50.

16. Paques M, Philippakis E, Falah S, et al. Indocyanine-green-guided targeted laser photocoagulation of capillary macroaneurysms in macular oedema: a pilot study. $\mathrm{Br} \mathrm{J}$ Ophthalmol 2017;101:170-174.

17. Grading diabetic retinopathy from stereoscopic color fundus photographs--an extension of the modified Airlie House classification. ETDRS report number 10. Early Treatment Diabetic Retinopathy Study Research Group. Ophthalmology 1991;98:786-806.

18. Hafner J, Salas M, Scholda C, et al. Dynamic Changes of Retinal Microaneurysms in Diabetes Imaged With In Vivo Adaptive Optics Optical Coherence Tomography. Invest Ophthalmol Vis Sci 2018;59:5932-5940.

19. Lammer J, Karst SG, Lin MM, et al. Association of Microaneurysms on Adaptive Optics Scanning Laser Ophthalmoscopy With Surrounding Neuroretinal Pathology and Visual Function in Diabetes. Invest Ophthalmol Vis Sci 2018;59:5633-5640.

20. Dubow M, Pinhas $A$, Shah N, et al. Classification of human retinal microaneurysms using adaptive optics scanning light ophthalmoscope fluorescein angiography. Invest Ophthalmol Vis Sci 2014;55:1299-309.

21. Stitt AW, Gardiner TA, Archer DB. Histological and ultrastructural investigation of retinal microaneurysm development in diabetic patients. Br J Ophthalmol 1995;79:362-7. 
22. Schmidt-Erfurth U, Lang GE, Holz FG, et al. RESTORE Extension Study Group.Three-year outcomes of individualized ranibizumab treatment in patients with diabetic macular edema: the RESTORE extension study. Ophthalmology 2014;121:1045-53.

23. Elman MJ, Ayala A, Bressler NM, et al. Diabetic Retinopathy Clinical Research Network. Intravitreal Ranibizumab for diabetic macular edema with prompt versus deferred laser treatment: 5-year randomized trial results. Ophthalmology 2015;122:375-81.

24. Brown DM, Schmidt-Erfurth U, Do DV, et al. Intravitreal Aflibercept for Diabetic Macular Edema: 100-Week Results from the VISTA and VIVID Studies. Ophthalmology 2015;122:204452.

25. Boyer DS, Nguyen QD, Brown DM, et al. RIDE and RISE Research Group. Outcomes with As-Needed Ranibizumab after Initial Monthly Therapy: Long-Term Outcomes of the Phase III RIDE and RISE Trials. Ophthalmology 2015;122:2504-13.

26. Korobelnik JF, Do DV, Schmidt-Erfurth U, et al. Intravitreal aflibercept for diabetic macular edema. Ophthalmology 2014;121:2247-54.

27. Boyer DS, Yoon YH, Belfort R Jr, et al; Ozurdex MEAD Study Group. Three-year, randomized, sham-controlled trial of dexamethasone intravitreal implant in patients with diabetic macular edema. Ophthalmology 2014;121:1904-14.

28. Sophie R, Lu N, Campochiaro PA. Predictors of Functional and Anatomic Outcomes in Patients with Diabetic Macular Edema Treated with Ranibizumab. Ophthalmology 2015;122(7):1395-401.

29. Shin YU, Hong EH, Lim HW, et al. Quantitative evaluation of hard exudates in diabetic macular edema after short-term intravitreal triamcinolone, dexamethasone implant or bevacizumab injections. BMC Ophthalmol 2017;17:182.

30. The Diabetic Retinopathy Clinical Research Network, Aflibercept, bevacizumab, or ranibizumab for diabetic macular edema. N Engl J Med 2015; 372:1193-120. 
31. Shin JY, Byeon SH, Kwon OW. Optical coherence tomography-guided selective focal laser photocoagulation: a novel laser protocol for diabetic macular edema. Graefes Arch Clin Exp Ophthalmol 2015;253:527-35.

32. Lee SN, Chhablani J, Chan CK, et al. Characterization of microaneurysm closure after focal laser photocoagulation in diabetic macular edema. Am J Ophthalmol 2013;155:905-12. 


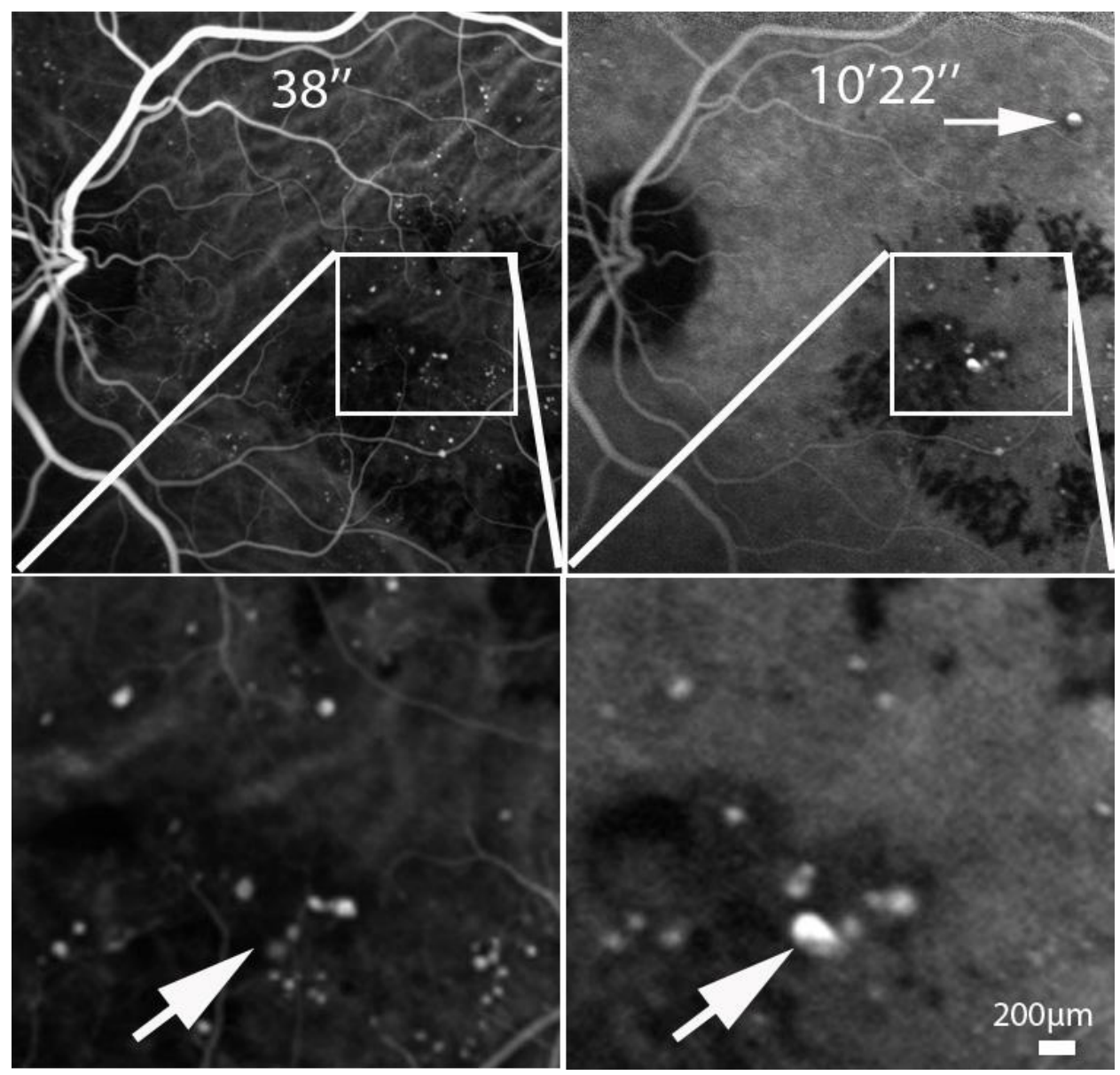

Supplementary figure 1. Comparison of ICG staining pattern of microaneurysms and TCs in case 10 (Same case as shown figure 4, bottom row). The time after dye injection is indicated. Between the early and late frame, note the fading contrast of the microaneurysms, while the TC increases contrast and size (arrow in magnification). Arrow in the upper right panel shows another TC not visible on early frames.

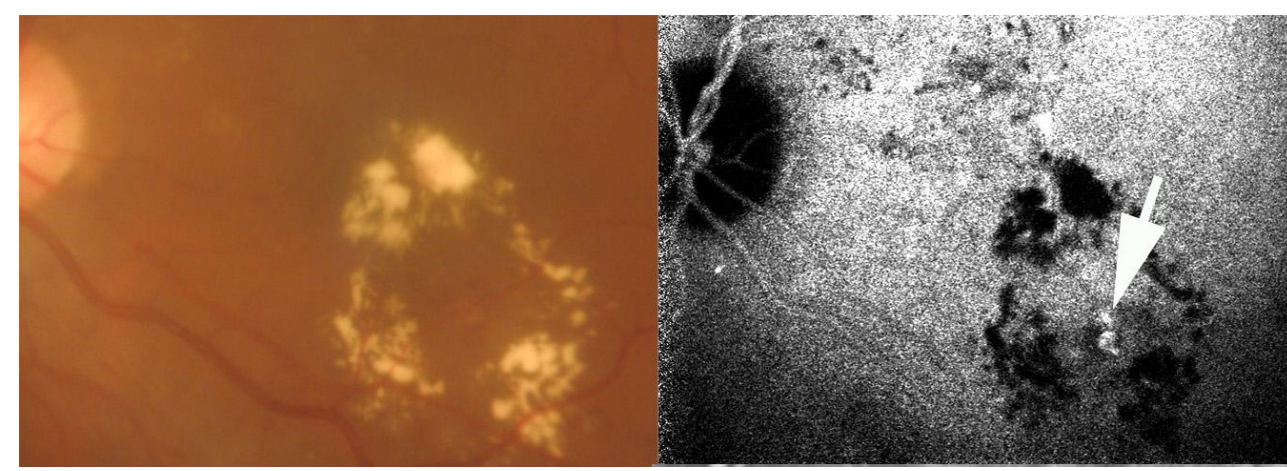

Supplementary figure 2. Case 3. Illustration of the presence of TCs (arrow) close to the geometrical center of a ring of hard exudates 